\title{
An Effective Technique to Identify Anomalous Accounts on Social Networks using Bloom Filter
}

\author{
Sarbjeet Kaur \\ Research Scholar \\ Computer Science \\ Engineering Department. \\ Universal Institute of \\ Engineering and Technology, Lalru. \\ Punjab Technical University, \\ Jalandhar, Punjab, India.
}

\author{
Prabhjot Kaur \\ Assistant Professor \\ Computer Science \\ Engineering Department. \\ Universal Institute of \\ Engineering and Technology, Lalru. \\ Punjab Technical University, \\ Jalandhar, Punjab, India.
}

\begin{abstract}
The anomaly detection is the technique which is applied to detect malicious activities from the social network data. The existing technique is based on to classify the Facebook accounts into three classes which are fake, genuine and moderate. To increase accuracy of account classification is increased when bloom filter is being applied in the algorithm. The bloom filter is the algorithm which learns from the previous experiences and drive new values. When the bloom filter is applied the accounts are classified into two classes. The simulation is being performed in MATLAB and it is being analyzed that accuracy is increased and execution time is reduced.
\end{abstract}

\section{General Terms}

Anomaly detection, Bloom Filter, Classification, Fake accounts, Online social Networks.

\section{Keywords}

Anomaly, Analysis, Classification.

\section{INTRODUCTION}

Social network analysis (SNA) is the process of investigating social structures using network and graph theories [1]. It portrays networked structures in terms of nodes (individual actors, people, or things within the network) and the ties, edges, or links (relationships or interactions) that connect them. Divisions of actors into clubs or "sub-groups" can be an essential part of social structure. It can be imperative in understanding how the network all in all is probably going to act. The idea of a clique is relatively simple. And no more general level, a clique is a sub-set of a network in which the actors are all the more closely and intensely tied to each other than they are to different members of the network. The strongest possible definition of a clique is some number of actors (more than two, usually three is used) who have every single possible tie present among themselves [2]. A Maximal Complete Sub-Graph is such a grouping, expanded to incorporate as numerous actors as possible. The N-clique approach tends to discover long and stringy groupings instead of the tight and discrete ones of the maximal approach. In some cases, $\mathrm{N}$-cliques can be found that have a property that is probably undesirable for some purposes: it is possible for members of $\mathrm{N}$-cliques to be connected by actors who are not, themselves, members of the clique. The sort of a restriction has the impact of forcing all paths among members of an nclique to happen by way of different members of the n-clique [3]. This approach is the N-Clan. The k-plex approach would seem to have a considerable amount in common with the nclique approach, yet k-plex analysis regularly gives a significant distinctive picture of the substructures of a graph.

An anomaly is defined as an unusual action showing an alternate behavior than others exhibit in a similar structure. The term additionally called an outlier, abnormality or special case, has been defined from numerous points of view by various authors. Anomaly detection is additionally viewed as similar to novelty detection in which already unobserved novel patterns in the data are detected [4]. They may at first appear to be same however in novelty detection upon the confirmation of new points they are for the most part incorporated into the model of normal behavior. The presence of anomalies in our data poses numerous problems which should be tackled deliberately. For instance, some kind of malicious users may construct a set of false identities and utilize them to communicate with an extensive random set of innocent users. Henceforth, detection of these anomalous activities in a network is a major concern as their presence may lead to heavy losses [5]. The anomalies are classified into chiefly three categories based upon the nature and scope of anomalies. Point anomalies, additionally known as global anomalies are found if a data object (i.e. a point) demonstrates an alternate behavior than that of the rest of the data. Also known as conditional anomalies, contextual anomalies are available in the data set if the data object deviates essentially with respect to a particular context [6]. Collective anomalies are encountered at whatever point a collection of data objects all in all depicts an alternate behavior than others, though the individual data objects may not be anomalous. Recently, another sort of anomaly, called horizontal anomaly has evolved in social networks which depict the presence of anomalies based upon the diverse sources of data available [7]. A dynamic anomaly exists with respect to past network behavior in which changes happen in the network with the passage of time. A static anomaly occurs with respect to remainder of the network ignoring the time factor. Labeled anomalies are identified with both structure of the network and the information gathered from vertex or edge attributes. Unlabeled anomalies are connected just to the network structure. No trait of a node or an edge is contemplated.

\section{LITERATURE REVIEW}

Weiling Chen, et.al (2016) proposed in this paper [8] that because of the potential damage the false information may be conveyed to the public. So, the false rumor detection has turned into a significant however challenging examination topic. In this paper, false rumors are viewed as anomalies and 
we perform Factor analysis of mixed data (FAMD) on our proposed features to detect these anomalies. Two strategies based on Euclidean separation and Cosine closeness are proposed to describe the deviation degree. The paper proposes two strategies to describe the deviation degree of Weibos to help detect the potential rumors. This paper shows that it can accomplish good performance and can shed light on automatic detection of false rumors on OSNs.

Cuneyt Gurcan Akcora, et.al (2014) proposed in this paper [9], the utilization of two snapshots from the Twitter network and dissects data interest patterns of users in time to understand individual and collective user behavior on social networks. It is seen that albeit more than $80 \%$ of all friendships on Twitter are made because of data interests, 83 $\%$ of all users have no less than one friendship that can be clarified neither by users' past interest nor collective behavior of other comparative users. The advantage of our approach is that it works notwithstanding when a user's tweet/bio data are not generally clean, robust and available.

Hamid Alipour, et.al (2015) proposed in this paper [10], an anomaly-based intrusion detection system is described for the IEEE 802.11 wireless networks based on behavioral analysis to detect deviations from normal behaviors that are triggered by wireless network attacks. This anomaly behavior analysis of the 802.11 protocols is based on monitoring the nconsecutive transitions of the convention state machine. This paper applies sequential machine learning techniques to show the n-transition patterns in the convention and characterize the probabilities of these transitions being normal. This paper has implemented several experiments to evaluate our system performance. By traversing two diverse wireless channels, the paper has accomplished a low false caution rate (under $0.1 \%$ ).

Flora Amato, et.al (2016) proposed that in this work [11], a general framework is introduced for event detection from Twitter. The framework aims to gather tweets related to a specific social event, keeping in mind the end goal to filter and classify those which can be relevant to detect malicious actions in Twitter communities. Relevant tweets are processed to raise an alert if there should be an occurrence of anomaly inside the collected set. The alert module implements a set of burst detection calculations so as to generate alerts at whatever point an event happens that means an anomaly in the broke down data stream is detected. The future work intends to provide the implementation of this system tuned with a domain related to the bioterrorism.

William Eberle, et.al (2015) proposed that in this work [12], an approach is displayed to process a stream of changes to the graph keeping in mind the end goal to productively identify any adjustments in the normative patterns and any adjustments in the anomalies to these normative patterns without processing every single past dat. The overall framework of our approach is called PLADS for Pattern Learning and Anomaly Detection in Streams. PLADS allows us to process information that is represented in data streams, discovering patterns and anomalies with minimal falsepositives, with a request of-magnitude accelerate over the traditional GBAD approach.

David Savage, et.al (2014) proposed that in this paper [13] the existing computational techniques are surveyed for detecting anomalies in online social networks. The paper characterizes anomalies as being either static or dynamic, and as being labeled or unlabeled, and survey methods for detecting these diverse types of anomalies. The paper suggests that the detection of anomalies in online social networks is composed of two sub-processes; the selection and calculation of network features, and the classification of observations from this feature space. Also, this paper provides an overview of the types of problems that anomaly detection can address and identifies key areas for future research. It is found that these diverse approaches can be helpfully ordered based on portrayal of anomalies as being static or dynamic and labeled or unlabeled.

\section{PROPOSED TECHNIQUE}

This work is based on to detect the fake Facebook accounts on the basis of activities of the users. In the existing technique, the formulas are applied on the basis of strength and no of accounts joined. The formulas applied will classify the accounts into fake, moderate and genuine. In this work, the improvement in the existing system is done in which bloom filter is applied which classify the data into fake and genuine accounts. Strength of nodes is calculated with summation of indegree and outdegree of nodes.

Calculation of trust score:

Trust Score $=\mathrm{Fa} / \mathrm{Za}$

Where, $\mathrm{Fa}$ is the number of friend request accepted by the nodes in the network which is being sent by the node ' $a$ ' and $\mathrm{Za}$ is the number of friend requests made by the node ' $\mathrm{a}$ '. The symbol ' $a$ ' represent any node in network.

Bloom filter is a type of probabilistic data structure which searches an item which is definitely in collection or not at all in collection [14]. Bloom filter is applied to both trust and strength of nodes in this work. Bloom filter finds a best value from all possible values after execution of no of iterations. The best value selected by bloom filter is used for classification of accounts as genuine or fake accounts class. This way bloom filter combination with trust and strength of nodes provides a more accurate results to classify accounts.

Algorithm 1:

Input: Facebook Account information

Output: Classification of accounts into two classes

1. A=Strength of account

2. $\mathrm{B}=$ Number of points joined

3. Calculate best value ()

4. $\mathrm{B}=$ Random point selected from the dataset

5. For $(\mathrm{i}=0 ; \mathrm{i}=\mathrm{n} ; \mathrm{i}++)$

6. Calculate best value $\mathrm{F}=\mathrm{F}(\mathrm{i}) / \mathrm{F}^{\prime}(\mathrm{i})$

7. end

8. If(best(i) $>$ best $(i+1)$

9. Best value $=$ best $(\mathrm{i})$

10. Else

11. Repeat step 5 to 9

12. Calculate accuracy

13. Accuracy=number of accounts classified /Total accounts

14. STOP

This algorithm is developed to classify the malicious accounts from online social networks. The code is implemented in 
MATLAB to obtain desired results after applying above calculations of trust score and strength. Best value is calculated from both trust score and strength to classify accounts into two classes that is fake and Genuine.

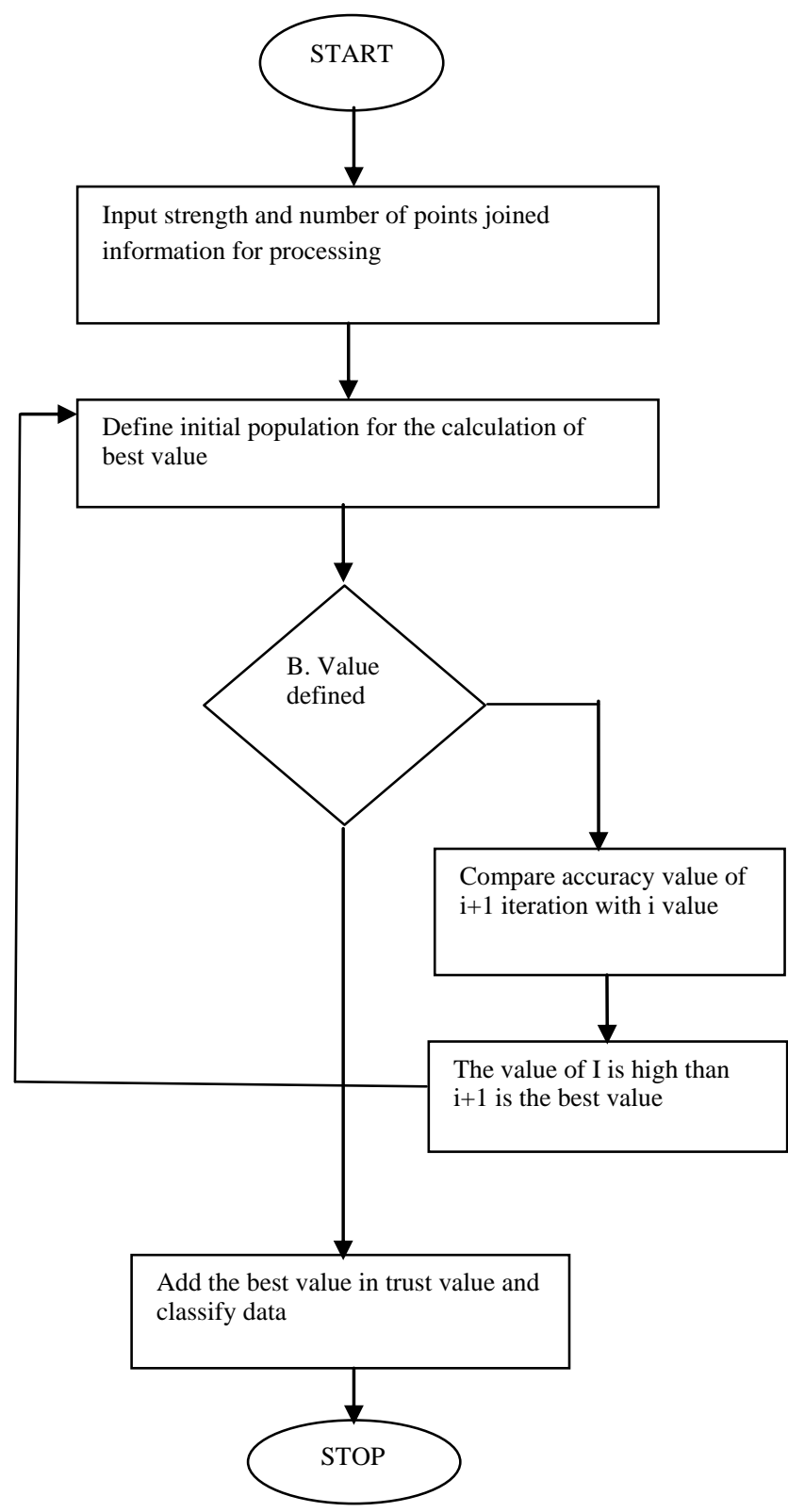

\section{RESULTS AND DISCUSSION}

The MATLAB is the tool which is used to perform simulation of proposed and existing models. The technique will be proposed which will be based on the bloom filter technique. In the technique of bloom filter the categorization the users into the two classed means the fake and genuine classes.

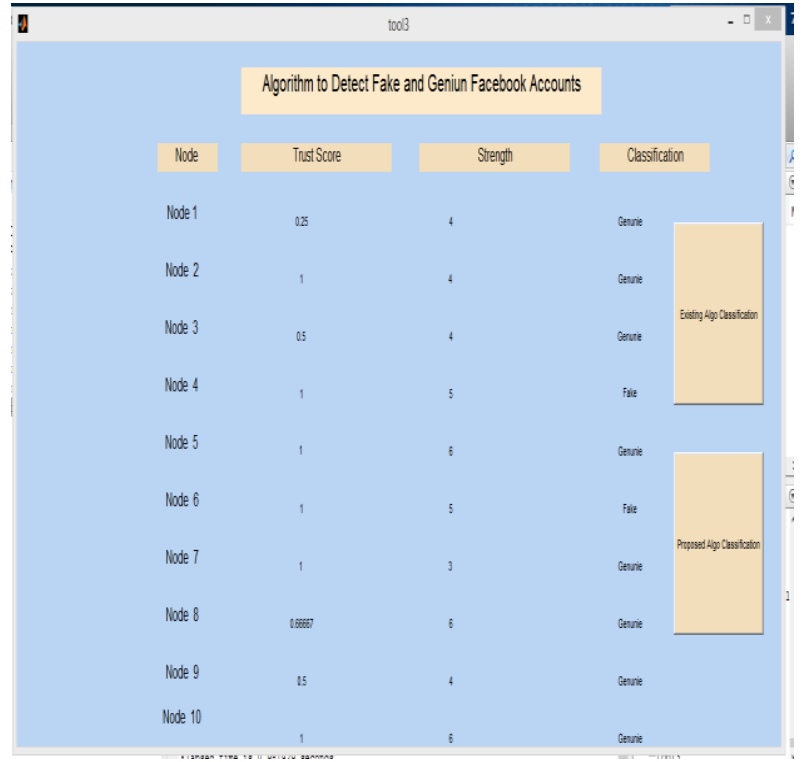

Fig. 2 Interface of implementation

As shown in the figure 2 , the interface is designed which classify the Facebook accounts as fake or genuine.

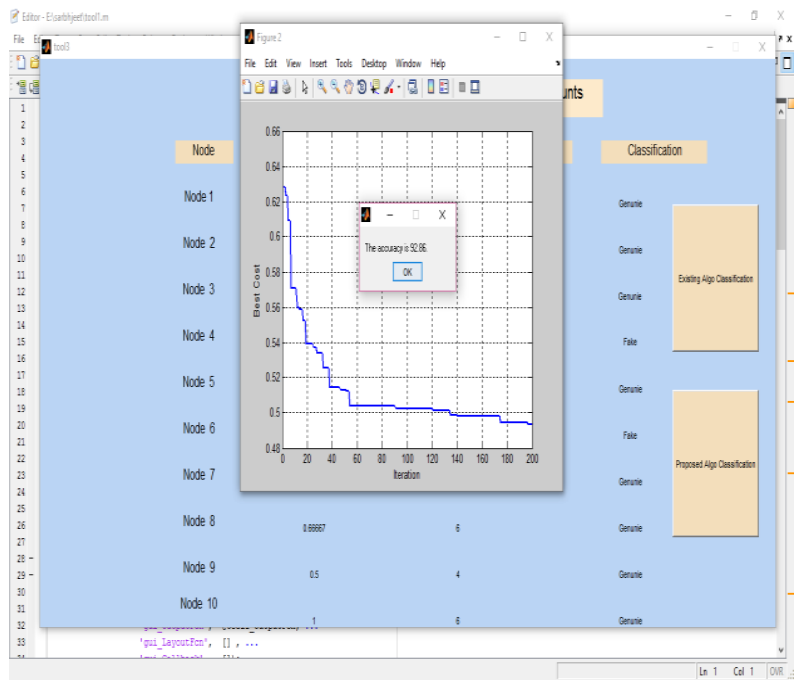

Fig. 3 Final result of proposed work

As shown in figure 3, final result of proposed work is represented by this interface.

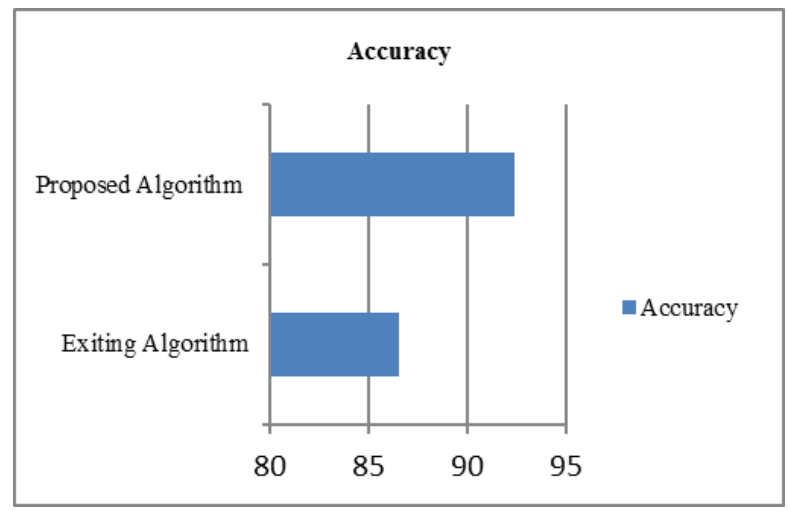

Fig. 4 Accuracy Comparison

As shown in figure 4, the accuracy of the proposed and existing algorithm is being compared and it is being analyzed 
that accuracy of proposed algorithm is increased to 92 percent from 86 percent.

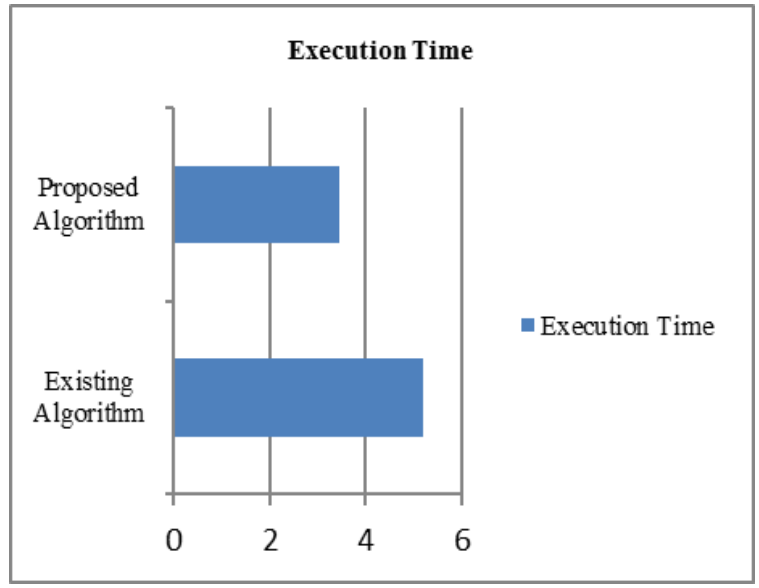

Fig. 5 Execution Time

As shown in figure 5, the execution time of the proposed algorithm is reduced as compared to existing algorithm.

\section{CONCLUSION AND FUTURE SCOPE}

In this work, it is being concluded that in the existing algorithm the social network accounts are classified into three classes which are fake, moderate and genuine. In this work, bloom filter is applied which can classify the accounts into two classes which are fake and genuine. The simulation is being performed in MATLAB and it is being analyzed that execution time is reduced and accuracy is increased as a result. In this work we used standard bloom filter. In future other parameters like use of scalable bloom filters can be used for more authentication

\section{REFERENCES}

[1] AbdolazimRezaei, ZarinahMohdKasirun, Vala Ali Rohani, TourajKhodadadi," Anomaly Detection in Online Social Networks Using Structure-Based Technique", The 8th International Conference for Internet Technology and Secured Transactions (ICITST-2013)

[2] Shota Saito, RyotaTomioka, Kenji Yamanishi," Early detection of persistent topics in social networks", 2015, Soc. Netw. Anal. Min, pp. 5:19

[3] Anita Zakrzewska and David A. Bader," A Dynamic Algorithm for Local Community Detection in Graphs",
2015 IEEE/ACM International Conference on Advances in Social Networks Analysis and Mining

[4] Renjun Hu, Charu C. Aggarwal, Shuai Ma, and Jinpeng Huai," An Embedding Approach to Anomaly Detection", 2016, IEEE

[5] Ravneet Kaur, Sarbjeet Singh," Detecting Anomalies in Online Social Networks using Graph Metrics", 2015, IEEE

[6] P. Kayalvizhi, C. AnoorSelvi,” Detecting Dynamic Topics in Social Network Using Citation based Anomaly Detection", 2015, IEEE Sponsored 9th International Conference on Intelligent Systems and Control (ISCO)

[7] Evangelos E. Papalexakis, Alex Beutel, Peter Steenkiste," Network Anomaly Detection using Co-clustering", 2012 IEEE/ACM International Conference on Advances in Social Networks Analysis and Mining

[8] Weiling Chen, Chai Kiat Yeo, Chiew Tong Lau, Bu Sung Lee," Behavior Deviation: An Anomaly Detection View of Rumor Preemption”, 2016, IEEE

[9] Cuneyt Gurcan Akcora, Barbara Carminati, Elena Ferrari, Murat Kantarcioglu," Detecting anomalies in social network data consumption", 2014, Soc. Network Analysis Min

[10] Hamid Alipour, Youssif B. Al-Nashif, Pratik Satam and Salim Hariri," Wireless Anomaly Detection based on IEEE 802.11 Behavior Analysis", 2015, IEEE

[11] Flora Amato, Giovanni Cozzolino, Antonino Mazzeo and Sara Romano," Detecting anomalies inTwitter stream for public security issues", 2016, IEEE

[12] William Eberle, Lawrence Holder," Streaming Data Analytics for Anomalies in Graphs", 2015, IEEE

[13] David Savage, Xiuzhen Jenny Zhang, Xinghuo Yu, Qingmai Wang," Anomaly Detection in Online Social Networks", 2014, Social Networks, Volume 39, pp. 6270, ISSN: 0378-8733

[14] Shahabeddin Geravand, Mahmood Ahmadi“Bloom filter application in network security: A-state-of-the-art-ofsurvey" 2013 Elsevier journal, computer networks, ScienceDirect. 